\title{
A First-Principles Study on Titanium-Decorated Adsorbent for Hydrogen Storage
}

\author{
Kai Ma ${ }^{1,2,3} \mathbb{D}^{\text {, Erfei Lv }}{ }^{1}$, Di Zheng ${ }^{1}$, Weichun Cui ${ }^{4, *}$, Shuai Dong ${ }^{1,2,3}$, Weijie Yang ${ }^{1,2,3}$, Zhengyang Gao ${ }^{1,2,3}$ \\ and Yu Zhou ${ }^{1}$
}

Citation: Ma, K.; Lv, E.; Zheng, D.; Cui, W.; Dong, S.; Yang, W.; Gao, Z.; Zhou, Y. A First-Principles Study on Titanium-Decorated Adsorbent for Hydrogen Storage. Energies 2021, 14 , 6845. https://doi.org/10.3390/ en14206845

Academic Editors: Adam Smoliński and Muhammad Aziz

Received: 4 August 2021

Accepted: 12 October 2021

Published: 19 October 2021

Publisher's Note: MDPI stays neutral with regard to jurisdictional claims in published maps and institutional affiliations.

Copyright: (c) 2021 by the authors. Licensee MDPI, Basel, Switzerland. This article is an open access article distributed under the terms and conditions of the Creative Commons Attribution (CC BY) license (https:/ / creativecommons.org/licenses/by/ $4.0 /)$.
1 Department of Power Engineering, North China Electric Power University, Baoding 071003, China; mak@ncepu.edu.cn (K.M.); lvef@ncepu.edu.cn (E.L.); 201702400431@ncepu.edu.cn (D.Z.); sdong@ncepu.edu.cn (S.D.); yangwj@ncepu.edu.cn (W.Y.); 51450690@ncepu.edu.cn (Z.G.); zhouyu@ncepu.edu.cn (Y.Z.)

2 Hebei Key Laboratory of Low Carbon and High Efficiency Power Generation Technology, North China Electric Power University, Baoding 071003, China

3 Baoding Key Laboratory of Low Carbon and High Efficiency Power Generation Technology, North China Electric Power University, Baoding 071003, China

4 Department of Law and Political Science, North China Electric Power University, Baoding 071003, China

* Correspondence: 51951918@ncepu.edu.cn

\begin{abstract}
Based on density functional theory calculation, we screened suitable Ti-decorated carbonbased hydrogen adsorbent structures. The adsorption characteristics and adsorption mechanism of hydrogen molecules on the adsorbent were also discussed. The results indicated that Ti-decorated double vacancy $(2 \times 2)$ graphene cells seem to be an efficient material for hydrogen storage. Ti atoms are stably embedded on the double vacancy sites above and below the graphene plane, with binding energy higher than the cohesive energy of Ti. For both sides of Ti-decorated double vacancy graphene, up to six $\mathrm{H}_{2}$ molecules can be adsorbed around each Ti atom when the adsorption energy per molecule is $-0.25 \mathrm{eV} / \mathrm{H}_{2}$, and the gravimetric hydrogen storage capacity is $6.67 \mathrm{wt} . \%$. Partial density of states (PDOS) analysis showed that orbital hybridization occurs between the $d$ orbital of the adsorbed $\mathrm{Ti}$ atom and $p$ orbital of $\mathrm{C}$ atom in the graphene layer, while the bonding process is not obvious during hydrogen adsorption. We expect that Ti-decorated double vacancy graphene can be considered as a potential hydrogen storage medium under ambient conditions.
\end{abstract}

Keywords: hydrogen storage; first-principles; graphene; titanium decoration; adsorption

\section{Introduction}

As an excellent substitute for fossil fuels, hydrogen is considered one of the most promising "green" fuels, because of its high heat value (144 MJ/kg), high energy density, abundant reserves, and the fact that it is environmental pollution free [1]. However, hydrogen storage performance is relatively poor; due to the low weight and volume densities of hydrogen adsorbed at ambient temperature, it cannot meet the strict parameters set by US Department of Energy (DOE). Therefore, the use of hydrogen as an energy carrier has not yet been commercialized, and the storage of hydrogen is currently the most important challenge $[2,3]$.

Traditional hydrogen storage methods use low-temperature and high-pressure liquid phase storage technology, which is difficult to apply at large scales due to high cost and safety problems [4]. Hydrogen storage in solid materials is considered to be a promising method that is safe, economical, and easy to transport. At present, common solid hydrogen storage materials can be divided into several categories: physical hydrogen storage such as metal-organic frameworks (MOFs), covalent-organic frameworks (COFs), carbon-based materials, chemical hydrogen storage materials like metal hydrides, and chemical hydrides.

The DOE has set strict targets for hydrogen storage systems, including that the weight and volumetric energy capacity of the systems must reach $2.2 \mathrm{kWh} / \mathrm{kg}(6.5 \mathrm{wt} . \%)$ and 
$\left.1.7 \mathrm{kWh} / \mathrm{L} \mathrm{(5} \mathrm{kg} \mathrm{H}_{2} / \mathrm{L}\right)$, respectively, and the minimum and maximum delivery temperature and pressure are -40 and $85^{\circ} \mathrm{C}$ and 5 and 12 bar, respectively [5]. To the best of our knowledge, there are currently no hydrogen storage materials that meet these stringent standards [6]. Therefore, the development of a novel high-capacity hydrogen storage material is currently an urgent need.

Because carbon-based materials possess the advantages of large specific surface area, numerous adsorption sites and a wide range of material sources, they are considered to be one of the candidates for hydrogen storage materials. Since Dillon et al. [7] proved the good hydrogen storage capacity of single-walled carbon nanotubes in 1997, a lot of studies on hydrogen storage using carbon-based materials have been conducted. Many carbon-based materials have demonstrated good hydrogen storage performance, including fullerene [8], carbon nanotubes [9-11], graphene [12-17], porous graphene [18-20], and zig-zag graphene $[21,22]$. Because of the high chemical energy barrier of the pristine carbon nanostructure, it does not suffice as a good hydrogen storage material [23]. Many studies have shown that the use of metal atoms to modify carbon nanostructures is an effective way of improving their hydrogen storage capacity [24]. Metals commonly used to decorate carbon backbones include transition metals [25-27], alkali metals [28], alkaline earth metals [29] and other simple metals [30]. First-principles calculations have attracted more and more attention because of their low cost, easy implementation, and ability to reveal mechanisms. Using first-principles calculations, many studies on hydrogen storage have achieved outstanding results. Zheng et al. [31] studied the hydrogen storage performance of lithium-decorated graphene materials and found a gravimetric hydrogen density of $3.8 \mathrm{wt} . \%$. Ramos-Castillo et al. [32] compared the hydrogen storage performance of $\mathrm{Ti}_{4}$ - and $\mathrm{Ni}_{4}$-doped defective graphene nanoplatelets. The results showed that $\mathrm{Ti}_{4}$-doped graphene offers better hydrogen storage performance, and its hydrogen gravimetric content can be as high as $3.4 \mathrm{wt} . \%$, while the gravimetric content of $\mathrm{Ni}_{4}$-doped graphene is only 0.30 wt. $\%$, 10-fold lower than $\mathrm{Ti}_{4}$-doped graphene. Liu et al. [33] provided evidence that the binding strength of hydrogen on Ti-decorated graphene could be significantly increased to 0.23-0.60 eV. Park et al. [34] found that the substitution of boron in graphene could enhance the adsorption energy of titanium, with the hydrogen storage capacity reaching as high as $7.9 \mathrm{wt} . \%$. Despite these meaningful results, there is still a common problem. They need to significantly reduce the storage temperature to achieve the required capacity, which is adverse to the requirements of practical applications that are close to environmental conditions [35].

In this study, we compare a variety of adsorbent structures by means of Ti modification, screening the most suitable adsorbent structure, and verifying the stability of the adsorbent from an energy point of view. Then, we research the adsorption characteristics of different numbers of hydrogen molecules on the adsorbent, thus obtaining the hydrogen storage capacity. Finally, we analyze the mechanism of hydrogen adsorption on the adsorbent by partial density of states.

\section{Computational Methods}

We performed all density functional theory (DFT) calculations with the Vienna Ab Initio Simulation Package (VASP). The exchange and correlation interactions were described using the generalized gradient approximation (GGA) in the form of the Perdew-BurkeErnzerhof (PBE) [35] and the projector-augmented-wave (PAW) potentials [36,37]. The cell shape and cell volume are not allowed to be changed, and all atoms are allowed to relax when the structure is optimized. The spin polarization and van der Waals interactions [38] were included in all calculations. During the structural optimization, the dipole correction is calculated parallel to the third lattice vector direction. The convergence standard is set to $10^{-5} \mathrm{eV}$ for the difference of the total energies of the last two consecutive steps and $0.05 \mathrm{eV} / \AA$ for the maximum force allowed on each atom. The graphene cells are separated by a vacuum region of $15 \AA$ to prevent the interactions of adjacent slabs. The energy cutoff was set as $450 \mathrm{eV}$ for the plane-wave basis. A $(6 \times 6 \times 1) \Gamma$-centered k-point grid for the 
Brillouin zone was used. Some of the main simulation parameters are presented in Table 1 below:

Table 1. Some mainly simulation parameters during calculation.

\begin{tabular}{cc}
\hline Calculation Details & Parameter \\
\hline spin polarization & ISPIN $=2$ \\
\hline energy cutoff $/ \mathrm{eV}$ & ENCUT $=450.0$ \\
\hline convergence condition (energy) $/ \mathrm{eV}$ & EDIFF $=1 \times 10^{-5}$ \\
\hline convergence condition (force) $/ \mathrm{eV} / \AA$ & $\mathrm{EDIFFG}=-0.05$ \\
\hline dipole correction & LDIPOL $=$ TRUE. \\
IDIPOL $=3$ \\
\hline degrees-of-freedom & ISIF $=2$ \\
\hline van der Waals interactions & IVDW $=12$ \\
\hline k-point & $6 \times 6 \times 1$ \\
\hline
\end{tabular}

The binding energy $\left(E_{b}\right)$ that can be used for determining the stability of the adsorbent is calculated by the following equation:

$$
E_{b}=\left(E_{\text {adsorbent }}-2 E_{\text {metal }}-E_{\text {graphene }}\right) / 2
$$

where $E_{\text {metal }}, E_{\text {graphene }}$ and $E_{\text {adsorbent }}$ are the energy of metal atom, graphene-based substrate and metal atom decorated substrate, respectively. On the basis of Equation (1), the binding energy is negative, indicating that metal atoms can be anchored on the graphene substrate. The more negative the binding energy, the more stable the binding. The binding energy should be less than the metal cohesive energy, avoiding the clustering of metal atoms [39].

To express the adsorption strength of hydrogen molecules, the adsorption energy $\left(E_{a d}\right)$ is calculated on the basis of Equation (2):

$$
E_{a d}=\left(E_{\text {system }}-E_{\text {adsorbent }}-n \times E_{\text {hydrogen }}\right) / n
$$

where $E_{\text {system }}$ and $E_{\text {hydrogen }}$ are the energy of the adsorption systems including hydrogen and adsorbent and a single hydrogen molecule, respectively; $n$ is the number of hydrogen molecules adsorbed.

The hydrogen storage capacity of a hydrogen storage system can be expressed by gravimetric storage capacity or gravimetric hydrogen density, which can be calculated using the following equation:

$$
g_{d}=\frac{M_{\text {hydrogen }}}{M_{\text {hydrogen }}+M_{\text {adsorbent }}} \times 100 \%
$$

where $g_{d}$ is the gravimetric hydrogen density, $M_{a d s o r b e n t}$ and $M_{\text {hydrogen }}$ are the mass of the adsorbent and all $\mathrm{H}_{2}$ molecules adsorbed, respectively.

\section{Results and Discussion}

As we discussed above, Ti-decorated carbon-based materials show good capability to adsorb hydrogen. Therefore, the first step is to screen the most suitable Ti-decorated carbon-based hydrogen adsorbent structures. Figure 1a shows the screening process. At the beginning, we researched the adsorption energies of different Ti-decorated carbon-based materials, like graphene and graphyne. After structural optimization, the structure of graphyne modified by Ti deforms significantly. Therefore, graphene is a better choice for the adsorbent substrate material. Then, we compared the binding energy of Ti atoms on different scales of graphene unit cells, including $(2 \times 2),(3 \times 3)$ and $(4 \times 4)$ cell. As studied in the existing literature $[17,22,24]$, in the Ti-decorated carbon-based materials, the amount 
of hydrogen molecules that can be absorbed by a Ti atom remained almost unchanged, while the mass of the $(3 \times 3)$ and $(4 \times 4)$ graphene cells was much higher than that of the $(2 \times 2)$ graphene cell. Therefore, the mass fraction of hydrogen adsorbed on a $(2 \times 2)$ cell of graphene was the largest, and the binding energy was the largest too, which is consistent with previous research [33]. Therefore, the $(2 \times 2)$ graphene cell was chosen as the size of the adsorbent substrate.

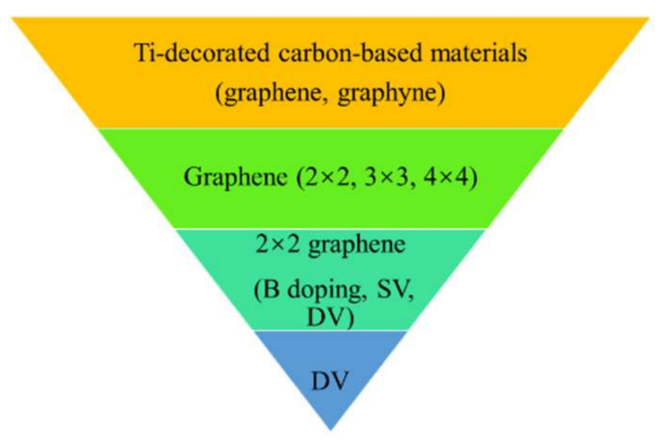

(a)

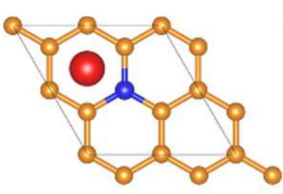

1B

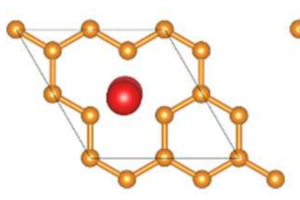

SV

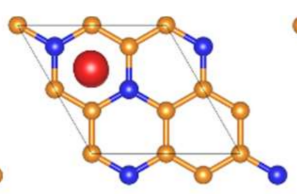

2B

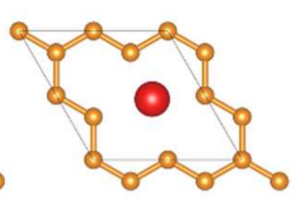

DV

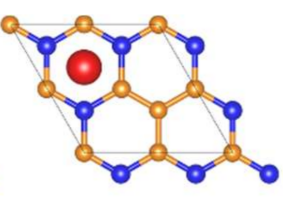

3B

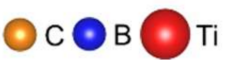

(b)

Figure 1. (a) The screening process for suitable adsorbent structure. Where B stands for boron, SV and DV stand for single vacancy defect and double vacancy defects graphene substrates, respectively; (b) the structure of different adsorbent substrates.

To suppress the clustering of $\mathrm{Ti}$ atoms, the binding energy of $\mathrm{Ti}$ atoms to the graphene substrates should be greater than the cohesive energy of Ti, which is $-4.85 \mathrm{eV}$. Several modifications were made to the graphene structure to improve the binding energy, including doping with one, two and three boron atoms, single vacancy defect (SV) and double vacancy defect (DV) graphene substrates. Figure 1b shows the structure of single vacancy graphene fragment, double vacancy graphene fragment, and different numbers of boron atoms doped graphene fragments.

The binding energy of $\mathrm{Ti}$ on different graphene structures are shown in Figure $2 \mathrm{a}$. From Figure $2 \mathrm{a}$, the binding energies of $\mathrm{Ti}$ with pristine graphene, one boron-doped graphene, two boron-doped graphene, and three boron-doped graphene are $-1.98 \mathrm{eV}$, $-3.08 \mathrm{eV},-3.68 \mathrm{eV},-4.73 \mathrm{eV}$, respectively, all of which are less than the cohesive energy of $\mathrm{Ti}$, implying that $\mathrm{Ti}$ atom prefers to aggregate on graphene under these cases. While in the cases of Ti-decorated SV and Ti-decorated DV graphene, the binding energies are $-5.51 \mathrm{eV}$ and $-6.70 \mathrm{eV}$, respectively, which means that the clustering of Ti atom on SV and DV graphene will not happen. Therefore, the hydrogen adsorption on Ti-decorated SV and Ti-decorated DV graphene will be calculated below.

As shown in Figure 2b, the adsorption behavior for two, four, six and eight $\mathrm{H}_{2}$ molecules adsorbed on both sides of Ti-decorated SV and DV graphene was studied. To be able to adsorb and desorb under environmental conditions, hydrogen adsorption should be in an intermediate state between physical adsorption and chemical adsorption. According to the results of previous studies, the adsorption energy should be between about -0.6 and about $-0.2 \mathrm{eV}$ [40]. In Figure $2 b$, the adsorption energies for two, four, six and eight $\mathrm{H}_{2}$ molecules adsorbed on $\mathrm{SV}$ graphene are all less than $-0.2 \mathrm{eV} / \mathrm{H}_{2}$, meaning that Ti-decorated SV graphene cannot satisfy the requirements of hydrogen recycling. For Ti-decorated DV graphene, when two, four and six $\mathrm{H}_{2}$ molecules are adsorbed on both sides of the substrate, the adsorption energies per molecule are $-0.31,-0.28$ and $-0.25 \mathrm{eV} / \mathrm{H}_{2}$, respectively, and these are suitable for reversible hydrogen storage media. In the case of eight $\mathrm{H}_{2}$ molecules adsorbed on both sides of the substrate, the adsorption energy is $-0.06 \mathrm{eV} / \mathrm{H}_{2}$, which is too low for hydrogen storage. Therefore, the maximum number for $\mathrm{H}_{2}$ molecules adsorbed on both sides of Ti-decorated DV graphene is six, 
with the gravimetric hydrogen density reaching up to $6.67 \mathrm{wt} . \%$, exceeding the technical indicators $(6.5 \mathrm{wt} . \%)$ issued by the DOE.

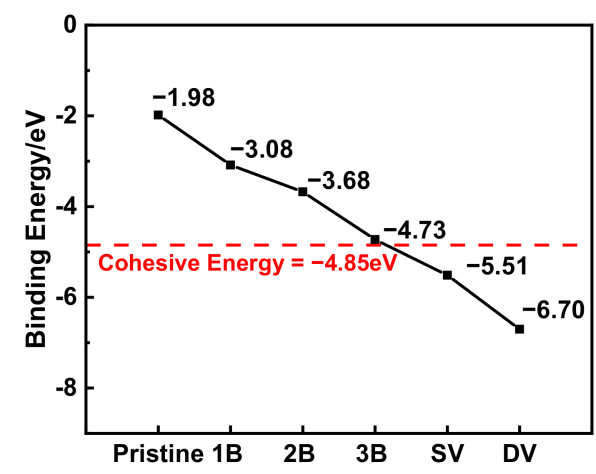

(a)

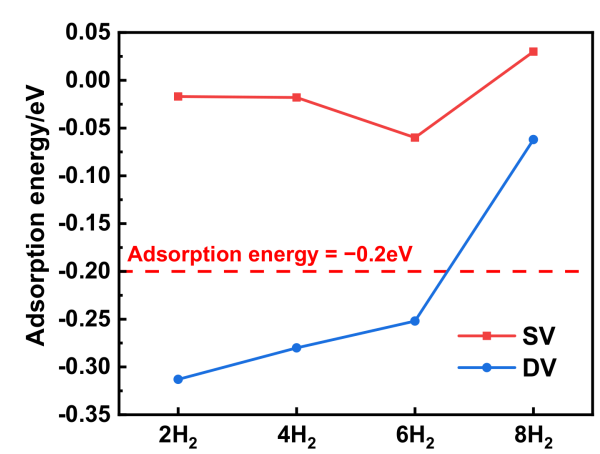

(b)

Figure 2. (a) The binding energy of Ti atoms on different graphene structures (Pristine means pristine $2 \times 2$ graphene cell, 1 B means one boron-doped graphene, 2 B means two boron-doped graphene, $3 \mathrm{~B}$ means three boron-doped graphene, respectively); (b) the adsorption energies for two, four, six and eight $\mathrm{H}_{2}$ molecules adsorbed on both sides of Ti-decorated SV and DV graphene.

Figure 3a,b show the side view and the top view of Ti-decorated DV graphene after structure optimization. Ti atoms prefer to be embedded on the double vacancy sites above and below the graphene plane approximately symmetrically. Since the radius of $\mathrm{Ti}$ atomis larger than the radius of the carbon atom, the metal atoms are displaced outward from the graphene surface [41]. The heights of Ti atoms above and below the graphene plane are 1.09 and $1.07 \AA$. The average Ti-C distances above and below the graphene plane are 2.19 and $2.18 \AA$. Figure $3 \mathrm{c}-\mathrm{f}$ show the side views of the equilibrium configuration for the adsorption of two, four, six and eight $\mathrm{H}_{2}$ on both sides of Ti-decorated DV graphene, respectively. Figure $3 g$ shows the top view of the equilibrium configurations for six $\mathrm{H}_{2}$ molecules absorbed on the Ti-decorated DV graphene.

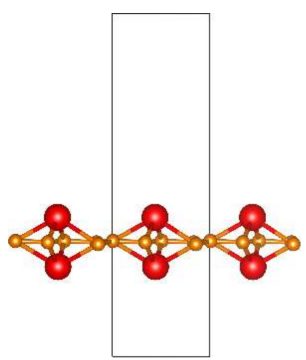

(a)

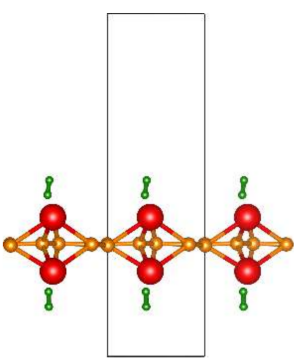

(b)

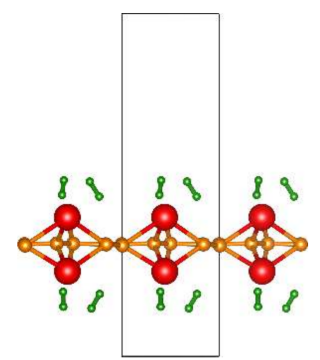

(c)

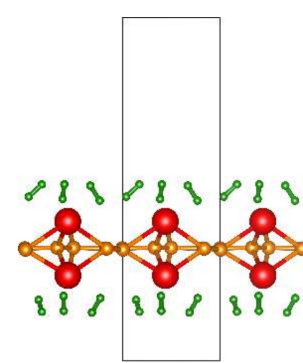

(d)

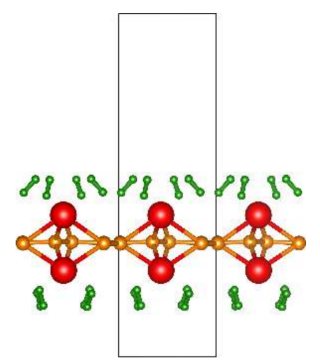

(e)

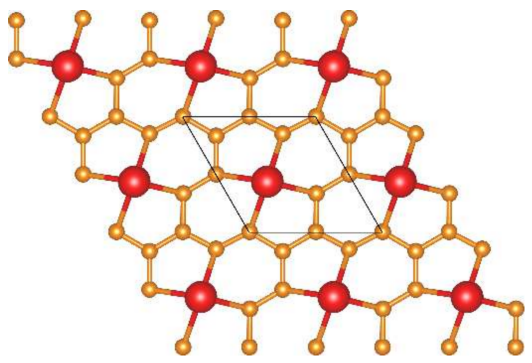

(f)

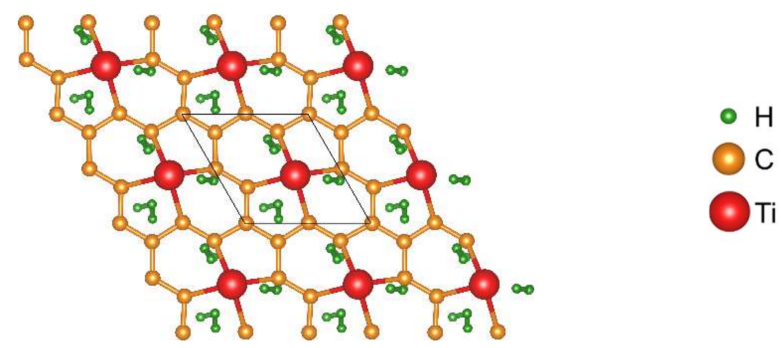

(g)

Figure 3. (a,b) The side view and top view of Ti-decorated DV graphene; $(\mathbf{c}-\mathbf{f})$ the side views of the equilibrium configurations of two, four, six and eight $\mathrm{H}_{2}$ molecules adsorbed on both sides of Ti-decorated DV graphene; (g) the top view of the configuration of six $\mathrm{H}_{2}$ absorbed on the Ti-decorated DV graphene. In the figure, green indicates $\mathrm{H}$ atoms, orange indicates $\mathrm{C}$ atoms and red indicates $\mathrm{Ti}$ atoms. 
The interaction between transition metals and hydrogen molecules has been described in previous research [42]. Hydrogen molecules provide charges to the unfilled $d$ orbitals of the transition metal atoms, while simultaneously obtaining electron filling antibonding orbitals from transition metal atoms. As a result, the bond of the hydrogen molecule is elongated. Table 2 shows the $\mathrm{H}-\mathrm{H}$ bond lengths and adsorption energies when two, four, six and eight $\mathrm{H}_{2}$ molecules are adsorbed, respectively. It can be seen that the $\mathrm{H}-\mathrm{H}$ bond length is stretched to the range $0.78-0.81 \AA$, which exceeds its value in free $\mathrm{H}_{2}(0.75 \AA)$ [33]. Moreover, when eight $\mathrm{H}_{2}$ molecules are adsorbed on the graphene, the adsorption energy is significantly lower than in other cases, and the change of the $\mathrm{H}-\mathrm{H}$ bond length is relatively small, indicating that $\mathrm{Ti}$ atoms have a weak adsorption effect on hydrogen. That is, the Ti-decorated DV graphene can only adsorb six $\mathrm{H}_{2}$ molecules at most.

Table 2. Calculated adsorption energies (eV) and bond lengths $(\AA)$ of $\mathrm{H}_{2}$ molecules when two, four, six and eight $\mathrm{H}_{2}$ molecules are adsorbed on Ti-decorated DV graphene, respectively.

\begin{tabular}{cccc}
\hline Number of $\mathbf{H}_{\mathbf{2}}$ & Adsorption Energy & Bond Length of H-H (Up) & Bond Length of H-H (Down) \\
\hline $2 \mathrm{H}_{2}$ & -0.3134 & 0.804 & 0.814 \\
\hline $4 \mathrm{H}_{2}$ & -0.2802 & 0.8040 .800 & 0.8040 .814 \\
\hline $6 \mathrm{H}_{2}$ & -0.2519 & 0.8040 .8000 .786 & 0.8040 .8140 .789 \\
\hline $8 \mathrm{H}_{2}$ & -0.0626 & 0.7880 .7830 .7840 .785 & 0.7890 .7840 .7820 .784 \\
\hline
\end{tabular}

To understand the synergistic interaction between $\mathrm{Ti}, \mathrm{H}$, and graphene, we further investigated the electronic structure figure of their chemical bonding. We first studied the interaction between the graphene layer and the adsorbed Ti atoms. Figure 4a shows the partial density of states (PDOS) of the relevant atomic orbitals of Ti-decorated DV graphene. In Figure $4 \mathrm{a}$, the DOS of the $d$ orbital of Ti is drawn with a red line, and the $p$ orbital of $C$ is drawn using a blue line. There is an obvious hybrid peak between $\operatorname{Ti}(d)$ and $C(p)$ near the Fermi level, indicating that there is a chemical bond between the Ti atom and DV graphene, and orbital hybridization contributes to the bonding process. It also proves the stability of the adsorbent structure. Figure $4 \mathrm{~b}$ displays the PDOS for the $d$ orbital of Ti and $s$ orbital of $\mathrm{H}$ when $\mathrm{H}_{2}$ molecules are adsorbed on the Ti-decorated DV graphene. There is no obvious orbital hybridization between $\mathrm{Ti}(d)$ and $\mathrm{H}(s)$ near the Fermi level, indicating that hydrogen adsorption mainly relies on charge transfer rather than bonding process, consistent with previous work [22].

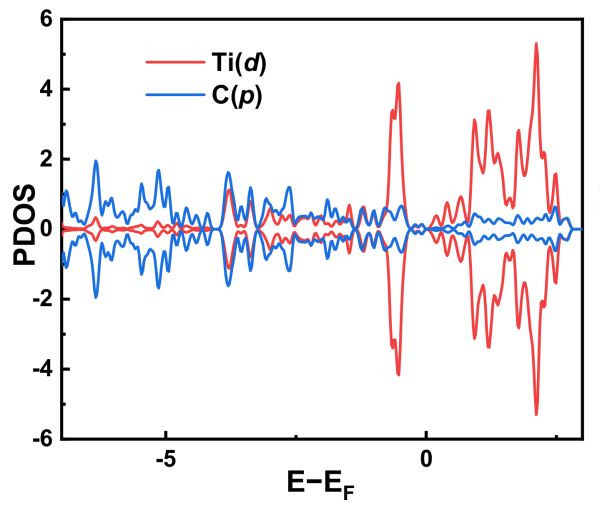

(a)

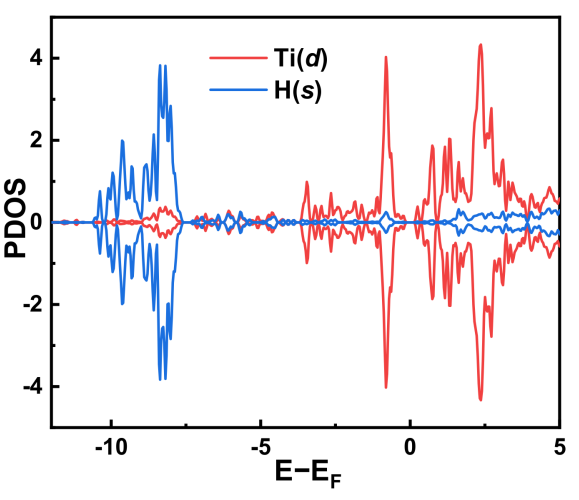

(b)

Figure 4. Partial density of states (PDOS) for (a) $d$ orbital of Ti (red line) and $p$ orbital of C (blue line) for Ti-decorated DV graphene; (b) $d$ orbital of Ti (red line) and $s$ orbital of $\mathrm{H}$ (blue line) when $\mathrm{H}_{2}$ are adsorbed on the Ti-decorated DV graphene. 


\section{Conclusions}

We performed DFT calculations to select the appropriate carbon-based hydrogen adsorbent, study the adsorption characteristics and adsorption mechanism of hydrogen molecules on the adsorbent. Among the adsorbents we analyzed, the Ti-decorated double vacancy $(2 \times 2)$ cell of graphene showed the best performance as an adsorbent, with a binding energy large enough to overcome the cohesive energy of Ti, avoiding the clustering of Ti atom on graphene surface. The adsorption energies per molecule are $-0.31,-0.28$ and $-0.25 \mathrm{eV} / \mathrm{H}_{2}$ when two, four and six $\mathrm{H}_{2}$ molecules are adsorbed on both sides of the substrate, respectively, satisfying the requirement that hydrogen molecules can be adsorbed and desorbed under ambient conditions while being conducive to practical application. The Ti-decorated DV graphene can adsorb six $\mathrm{H}_{2}$ molecules on both sides at most, with gravimetric storage capacity up to $6.67 \mathrm{wt} . \%$. There is a bonding process between Ti atoms and DV graphene, while hydrogen adsorption mainly relies on charge transfer rather than a bonding process. We expect that Ti-decorated double vacancy graphene can be regarded as a potential high-capacity hydrogen storage medium.

Author Contributions: Conceptualization, K.M. and E.L.; Data curation, D.Z.; Formal analysis, E.L. and D.Z.; Funding acquisition, W.Y. and Y.Z.; Investigation, K.M. and Z.G.; Methodology, D.Z. and W.C.; Project administration, W.C.; Resources, W.C.; Software, K.M.; Supervision, S.D. and Z.G.; Validation, S.D. and Z.G.; Writing-original draft, K.M. and E.L.; Writing—review \& editing, W.Y. All authors have read and agreed to the published version of the manuscript.

Funding: This research was funded by the National Natural Science Foundation of China (funding number:52006073), Natural Science Foundation of Hebei (funding number: E2020502023), and the Fundamental Research Funds for the Central Universities (funding number: 2021 MS121).

Institutional Review Board Statement: Not applicable.

Informed Consent Statement: Not applicable.

Data Availability Statement: Not applicable.

Conflicts of Interest: The authors declare no conflict of interest. The funders had no role in the design of the study; in the collection, analyses, or interpretation of data; in the writing of the manuscript, or in the decision to publish the results.

\section{References}

1. Schlapbach, L.; Züttel, A. Hydrogen-storage materials for mobile application. Nature 2001, 414, 353-358. [CrossRef]

2. Züttel, A. Materials for hydrogen storage. Mater. Today 2003, 6, 24-33. [CrossRef]

3. Ding, Z.; Yang, W.; Huo, K.; Shaw, L. Thermodynamics and Kinetics Tuning of LiBH4 for Hydrogen Storage. Prog. Chem. 2020, 64. [CrossRef]

4. Andersson, J.; Grönkvist, S. Large-scale storage of hydrogen. Int. J. Hydrogen Energy 2019, 44, 11901-11919. [CrossRef]

5. U.S. Department of Energy. 2017. Available online: www.energy.gov/eere/fuelcells/hydrogen-storage (accessed on 8 June 2021).

6. Jena, P. Materials for hydrogen storage: Past, present, and future. J. Phys. Chem. Lett. 2011, 2, 206-211. [CrossRef]

7. Dillon, A.C.; Jones, K.M.; Bekkedahl, T.A.; Kiang, C.H.; Bethune, D.S.; Heben, M.J. Storage of hydrogen in single-walled carbon nanotubes. Nature 1997, 386, 377-379. [CrossRef]

8. Yoon, M.; Yang, S.; Hicke, C.; Wang, E.; Geohegan, D.; Zhang, Z. Calcium as the superior coating metal in functionalization of carbon fullerenes for high-capacity hydrogen storage. Phys. Rev. Lett. 2008, 100, 206806. [CrossRef]

9. Lee, H.; Ihm, J.; Cohen, M.L.; Louie, S.G. Calcium-decorated carbon nanotubes for high-capacity hydrogen storage: First-principles calculations. Phys. Rev. B 2009, 80, 115412. [CrossRef]

10. López-Corral, I.; Germán, E.; Juan, A.; Volpe, M.A.; Brizuela, G.P. DFT study of hydrogen adsorption on palladium decorated graphene. J. Phys. Chem. C 2011, 115, 4315-4323. [CrossRef]

11. Xiao, H.; Li, S.H.; Cao, J.X. First-principles study of Pd-decorated carbon nanotube for hydrogen storage. Chem. Phys. Lett. 2009, 483, 111-114. [CrossRef]

12. Ao, Z.M.; Jiang, Q.; Zhang, R.Q.; Tan, T.T.; Li, S. Al doped graphene: A promising material for hydrogen storage at room temperature. J. Appl. Phys. 2009, 105, 074307. [CrossRef]

13. Ataca, C.; Akturk, E.; Ciraci, S.; Ustunel, H. High-capacity hydrogen storage by metallized graphene. Appl. Phys. Lett. 2008, 93, 043123. [CrossRef]

14. Ataca, C.; Aktürk, E.; Ciraci, S. Hydrogen storage of calcium atoms adsorbed on graphene: First-principles plane wave calculations. Phys. Rev. B 2009, 79, 041406. [CrossRef] 
15. Tozzini, V.; Pellegrini, V. Prospects for hydrogen storage in graphene. Phys. Chem. Chem. Phys. 2013, 15, 80-89. [CrossRef]

16. Liang, X.; Ng, S.-P.; Ding, N.; Wu, C.-M.L. Strain-induced switch for hydrogen storage in cobalt-decorated nitrogen-doped graphene. Appl. Surf. Sci. 2019, 473, 174-181. [CrossRef]

17. Chu, S.; Hu, L.; Hu, X.; Yang, M.; Deng, J. Titanium-embedded graphene as high-capacity hydrogen-storage media. Int. J. Hydrog. Energy 2011, 36, 12324-12328. [CrossRef]

18. Jain, V.; Kandasubramanian, B. Functionalized graphene materials for hydrogen storage. J. Mater. Sci. 2019, 55, 1865-1903. [CrossRef]

19. Ao, Z.M.; Dou, S.X.; Xu, Z.M.; Jiang, Q.G.; Wang, G.X. Hydrogen storage in porous graphene with Al decoration. Int. J. Hydrog. Energy 2014, 39, 16244-16251. [CrossRef]

20. Wang, F.; Zhang, T.; Hou, X.; Zhang, W.; Tang, S.; Sun, H.; Zhang, J. Li-decorated porous graphene as a high-performance hydrogen storage material: A first-principles study. Int. J. Hydrog. Energy 2017, 42, 10099-10108. [CrossRef]

21. Wu, M.; Gao, Y.; Zhang, Z.; Zeng, X.C. Edge-decorated graphene nanoribbons by scandium as hydrogen storage media. Nanoscale 2012, 4, 915-920. [CrossRef]

22. Lebon, A.; Carrete, J.; Gallego, L.J.; Vega, A. Ti-decorated zigzag graphene nanoribbons for hydrogen storage. A van der Waals-corrected density-functional study. Int. J. Hydrog. Energy 2015, 40, 4960-4968. [CrossRef]

23. Chan, S.-P.; Chen, G.; Gong, X.G.; Liu, Z.-F. Chemisorption of hydrogen molecules on carbon nanotubes under high pressure. Phys. Rev. Lett. 2001, 87, 205502. [CrossRef]

24. Reunchan, P.; Jhi, S.-H. Metal-dispersed porous graphene for hydrogen storage. Appl. Phys. Lett. 2011, 98, 093103. [CrossRef]

25. Yuan, L.; Wang, D.; Gong, J.; Zhang, C.; Zhang, L.; Zhang, M.; Wu, X.; Kang, L. First-principles study of V-decorated porous graphene for hydrogen storage. Chem. Phys. Lett. 2019, 726, 57-61. [CrossRef]

26. Chakraborty, B.; Modak, P.; Banerjee, S. Hydrogen storage in yttrium-decorated single walled carbon nanotube. J. Phys. Chem. C 2012, 116, 22502-22508. [CrossRef]

27. Yildirim, T.; Ciraci, S. Titanium-decorated carbon nanotubes as a potential high-capacity hydrogen storage medium. Phys. Rev. Lett. 2005, 94, 175501. [CrossRef] [PubMed]

28. Lu, R.; Rao, D.; Meng, Z.; Zhang, X.; Xu, G.; Liu, Y.; Kan, E.; Xiao, C.; Deng, K. Boron-substituted graphyne as a versatile material with high storage capacities of $\mathrm{Li}$ and $\mathrm{H}_{2}$ : A multiscale theoretical study. Phys. Chem. Chem. Phys. 2013, 15, 16120-16126. [CrossRef]

29. Hussain, T.; Pathak, B.; Ramzan, M.; Maark, T.A.; Ahuja, R. Calcium doped graphane as a hydrogen storage material. Appl. Phys. Lett. 2012, 100, 183902. [CrossRef]

30. Ao, Z.M.; Peeters, F.M. High-capacity hydrogen storage in Al-adsorbed graphene. Phys. Rev. B 2010, 81, 205406. [CrossRef]

31. Zheng, N.; Yang, S.; Xu, H.; Lan, Z.; Wang, Z.; Gu, H. A DFT study of the enhanced hydrogen storage performance of the Li-decorated graphene nanoribbons. Vacuum 2020, 171, 109011. [CrossRef]

32. Ramos-Castillo, C.M.; Reveles, J.U.; Cifuentes-Quintal, M.E.; Zope, R.R.; de Coss, R. Ti4- and Ni4-Doped Defective Graphene Nanoplatelets as Efficient Materials for Hydrogen Storage. J. Phys. Chem. C 2016, 120, 5001-5009. [CrossRef]

33. Liu, Y.; Ren, L.; He, Y.; Cheng, H.P. Titanium-decorated graphene for high-capacity hydrogen storage studied by density functional simulations. J. Phys. Condens. Matter 2010, 22, 445301. [CrossRef]

34. Park, H.L.; Yoo, D.S.; Yi, S.C.; Chung, Y.C. Theoretical investigation of Ti-adsorbed graphene for hydrogen storage using the ab-initio method. J. Nanosci. Nanotechnol. 2011, 11, 6131-6135. [CrossRef] [PubMed]

35. Perdew, J.P.; Burke, K.; Ernzerhof, M. Generalized Gradient Approximation Made Simple. Phys. Rev. Lett. 1996, 77, 3865-3868. [CrossRef] [PubMed]

36. Kresse, G.; Furthmüller, J. Efficient iterative schemes for ab initio total-energy calculations using a plane-wave basis set. Phys. Rev. B 1996, 54, 11169-11186. [CrossRef]

37. Kresse, G.; Furthmüller, J. Efficiency of ab-initio total energy calculations for metals and semiconductors using a plane-wave basis set. Comput. Mater. Sci. 1996, 6, 15-50. [CrossRef]

38. Grimme, S.; Antony, J.; Ehrlich, S.; Krieg, H. A consistent and accurate ab initio parametrization of density functional dispersion correction (DFT-D) for the 94 elements H-Pu. J. Chem. Phys. 2010, 132, 154104. [CrossRef] [PubMed]

39. Yang, W.; Xu, S.; Ma, K.; Wu, C.; Gates, I.D.; Ding, X.; Meng, W.; Gao, Z. Geometric structures, electronic characteristics, stabilities, catalytic activities, and descriptors of graphene-based single-atom catalysts. Nano Mater. Sci. 2020, 2, 120-131. [CrossRef]

40. Yoon, M.; Yang, S.; Wang, E.; Zhang, Z. Charged fullerenes as high-capacity hydrogen storage media. Nano Lett. 2007, 7, 2578-2583. [CrossRef]

41. Krasheninnikov, A.V.; Lehtinen, P.O.; Foster, A.S.; Pyykko, P.; Nieminen, R.M. Embedding transition-metal atoms in graphene: Structure, bonding, and magnetism. Phys. Rev. Lett. 2009, 102, 126807. [CrossRef]

42. Kubas, G.J. Metal-dihydrogen and $\sigma$-bond coordination: The consummate extension of the Dewar-Chatt-Duncanson model for metal-olefin $\pi$ bonding. J. Organomet. Chem. 2001, 635, 37-68. [CrossRef] 